\section{To the paper:}

\section{McCarthy et al.: What are appropriate initial and salvage therapies for patients with thrombotic thrombocytopenic purpura (TTP)?}

Thrombotic thrombocytopenic purpura [TTP], is rather rare (its occurrence being 1:1 million) but extremely interesting disease from several viewpoints:

- Its pathogenesis: new discoveries are made practically yearly in the field so that the main pathogenetic pathways, which were dark and not understood twenty years ago, are now known

- Diagnosis of the disease is another interesting chapter. It is well known that classical pentad of symptoms is not necessary any more for making a diagnosis. Important new laboratory tests were introduced into diagnostic process

- Genetic changes were discovered

- Clinical types were specified - not only basic differentiation as inborn or acquired disease with their specific diagnostic symptoms but also specifications of predisposing factors to relapses are investigated

- Even though much attention has been given to the disease recently, there is no finished randomized controlled study. Physicians rely on their own experience or on reports dealing with few patients only. Usually, no standard set of diagnostic and therapeutic tools is used in these reports, which makes it difficult to compare them in therapeutic decisions

- Progress in therapy is of enormous significance in clinical practice. It can be (with a bit of simplification) stated that the disease, which was in $90 \%$ lethal for often young patients 25 years ago is today in $90 \%$ treatable. With these quick developments in therapy, this disease has become attractive in clinical practice

In the report, authors present their experience with one of the largest cohorts of treated patients. Their concise presentation in which they point out major problems in diagnosis and therapy, is to be admired:

- As to the diagnosis: I would more than agree with the authors' statement, that there is no need for the classical pentad to be present for diagnosis. At the same time, though, I also feel, in agreement with the authors, that the presence of anemia and thrombocytopenia only is not enough to start treatment, which can be more detrimental than beneficial if the definitive diagnosis proves not to be TTP

- As to therapy: this is the most important and well-presented part of the article. Therapy is to be started within 6 hours in acute cases. It is not easy both diagnostically and technically: It is necessary to have diagnostic service and qualified personnel available round the clock. Proper equipment is necessary with adequate cell separators in place. In our teaching hospital, we generally start treating the patient within the same time frame if not earlier. We see this as necessary for satisfactory clinical outcome.

The therapeutic options are very clearly put in the article due to authors' extensive experience. There is a number of reports in the literature listing just the therapeutic options without a clear conclusion for quick orientation at patient's bedside. The major demand is plasma exchange (1 plasma volume exchange per day) and administration of corticosteroids (prednisone $1 \mathrm{mg} / \mathrm{kg}$ body weight). That is in agreement with our own experience. It is important to note that SD plasma is to be preferred as replacement.

The importance of salvage therapy in cases where the initial therapy with plasma exchange and immune suppression has failed or in cases of relapsed disease is pointed out. The question of splenectomy and its timing is still under discussion. Those who have their own experience with TTP could confirm how difficult it is to decide if splenectomy should be performed or whether there is still an option of another treatment modality. Theoretical explanation of the significance of splenecomy is one of the important points in the report. This is often omitted in literature. Since the spleens were examined histologically and histochemically, intraspelnic destruction of platelets could be shown.

In conclusion I would like to state that this report concisely presents main and most important facts about this rare but severe disease.

Prof. MUDr. Milan Bláha,CSc., University Hospital in Hradec Králové, II ${ }^{\text {nd }}$ Department of Internal Medicine, 50005 Hradec Králové, Czech Republic. e-mail: blaha@fnhk.cz 